\title{
Multiplicity of Solutions for a Sublinear Quasilinear Schrödinger Equation
}

\author{
Gui Bao* and Tingzhi Cheng
}

\begin{abstract}
In this paper, we are concerned with the multiplicity of solutions for a class of quasilinear elliptic equation arising from plasma physics. By using a dual approach, the existence of infinitely many small solutions are obtained. As a main novelty with respect to some previous results, we assume the potential $V$ may changes sign and do not require any condition at infinity on the nonlinear term.
\end{abstract}

\section{Introduction}

This paper is concerned with the multiplicity of solutions for a quasilinear Schrödinger equation of the form

$$
-\Delta u+V(x) u-\Delta\left(u^{2}\right) u=g(x, u), \quad x \in \mathbb{R}^{N},
$$

where $N=3,4$ and the potential $V(x)$ may change sign.

The quasilinear Schrödinger equation arises in several models of different physical phenomena, such as in the study of superfluid films in plasma physics, in condensed matter theory, etc (see [1,2 and the references therein). Recently, (1.1) has been extensively studied by many authors, we can for instance cite $3,8,10,15,17]$ and the references therein. For example, in [15], by using a constrained minimization argument, the existence of positive ground state solution was proved by Poppenberg, Schmitt and Wang. In 12, by a change of variables, Liu, Wang and Wang used an Orlicz space to prove the existence of soliton solution via mountain pass theorem. In [3], Colin and Jeanjean also made use of change variables to prove the existence of positive solution in the Sobolev space $H^{1}\left(\mathbb{R}^{N}\right)$. The same method of changing variables was also used recently to obtain positive solutions for the case of critical growth (see [4]). There are also some papers investigating the sublinear case. In [17], Zhou and Wu studied (1.1) with $V(x)=0$ in a bounded domain. Liang, Gao and Li [10] treated a quasilinear Schrödinger equation with $0<\alpha \leq V(x) \leq \gamma<\infty$.

In the present paper, without imposing any growth condition of $g(x, u)$ at infinity with respect to $u$, the existence of infinitely many small solutions for the problem (1.1)

Received December 1, 2018; Accepted October 30, 2019.

Communicated by Eiji Yanagida.

2010 Mathematics Subject Classification. 35J20, 35J62.

Key words and phrases. quasilinear, Schrödinger equation, dual approach, sublinear.

*Corresponding author. 
is obtained. Furthermore, unlike [10,17, the potential $V$ in this paper is allowed to be sign-changing.

We introduce the following hypotheses for the problem (1.1).

(V) $V \in C\left(\mathbb{R}^{N}, \mathbb{R}\right), \inf _{x \in \mathbb{R}^{N}} V(x)>-\infty$ and for each $M>0$, meas $\left\{x \in \mathbb{R}^{N}, V(x) \leq\right.$ $M\}<+\infty$, where meas denotes the Lebesgue measure in $\mathbb{R}^{N}$.

$\left(\mathrm{G}_{1}\right)$ There exists a constant $\delta_{1}>0$ such that $g(x,-t)=-g(x, t)$ for all $|t| \leq \delta_{1}$ and all $x \in \mathbb{R}^{N}$.

$\left(\mathrm{G}_{2}\right) \lim _{t \rightarrow 0} \frac{g(x, t)}{t}=+\infty$ uniformly for $x \in \mathbb{R}^{N}$.

$\left(\mathrm{G}_{3}\right)$ There exist constants $\delta_{2}>0$ and $0<s<1$ such that $g \in C\left(\mathbb{R}^{N} \times\left[-\delta_{2}, \delta_{2}\right], \mathbb{R}\right)$ and

$$
|g(x, t)| \leq b(x)|t|^{s}, \quad|t| \leq \delta_{2}, \quad \forall x \in \mathbb{R}^{N}
$$

where $b(x) \in L^{2 /(1-s)}\left(\mathbb{R}^{N}\right)$.

Our main result for $(1.1)$ is the following theorem.

Theorem 1.1. Suppose that $N=3,4$ and $(\mathrm{V}),\left(\mathrm{G}_{1}\right)-\left(\mathrm{G}_{3}\right)$ hold. Then the problem (1.1) has infinitely many solutions converging to zero.

Throughout this paper, $C>0$ denotes various positive constants which are not essential to our problem and may change from line to line.

\section{Preliminaries and useful lemmas}

Let $L^{p}(\Omega), 1 \leq p \leq+\infty, \Omega \subseteq \mathbb{R}^{N}$ denotes a Lebesgue space, the norm in $L^{p}(\Omega)$ is denoted by $|\cdot|_{p, \Omega}$. Let $H_{0}^{1}(\Omega), \Omega \subset \mathbb{R}^{N}$, and $H^{1}\left(\mathbb{R}^{N}\right)$ denote the usual Sobolev spaces. From $(\mathrm{V})$, we know that there exist constants $V_{0}>0$ and $\sigma>0$ such that $\widetilde{V}(x):=V(x)+V_{0}>\sigma$ for all $x \in \mathbb{R}^{N}$.

Set

$$
\begin{gathered}
E(\Omega)=\left\{u \in H_{0}^{1}(\Omega): \int_{\Omega}\left(|\nabla u|^{2}+\widetilde{V}(x) u^{2}\right) d x<+\infty\right\}, \\
E=E\left(\mathbb{R}^{N}\right)=\left\{u \in H^{1}\left(\mathbb{R}^{N}\right): \int_{\mathbb{R}^{N}}\left(|\nabla u|^{2}+\widetilde{V}(x) u^{2}\right) d x<+\infty\right\}
\end{gathered}
$$

with the norms

$$
\begin{gathered}
\|u\|_{\Omega}=\|u\|_{E, \Omega}=\left(\int_{\Omega}\left(|\nabla u|^{2}+\widetilde{V}(x) u^{2}\right) d x\right)^{1 / 2} \\
\|u\|=\|u\|_{E, \mathbb{R}^{N}}=\left(\int_{\mathbb{R}^{N}}\left(|\nabla u|^{2}+\widetilde{V}(x) u^{2}\right) d x\right)^{1 / 2}
\end{gathered}
$$


respectively. Denote $2^{*}=2 N /(N-2)$ for $N \geq 3$. By the continuity of the embedding $E(\Omega) \hookrightarrow L^{r}(\Omega), r \in\left[2,2^{*}\right], \Omega \subseteq \mathbb{R}^{N}$, there exist constants $\tau_{r}>0,2 \leq r \leq 2^{*}$ such that $|u|_{r, \Omega} \leq \tau_{r}\|u\|_{E, \Omega}, \forall u \in E(\Omega)$. Moreover, similar to Lemma 3.4 in [18], we may prove that under the assumption $(\mathrm{V})$, the embedding $E(\Omega) \hookrightarrow L^{r}(\Omega)$ is compact for $2 \leq r<2^{*}$, $\Omega \subseteq \mathbb{R}^{N}$.

Let $\widetilde{g}(x, u)=g(x, u)+V_{0} u$. We may seek to obtain solutions of (1.1) by looking for critical points of the associated functional $J: H^{1}\left(\mathbb{R}^{N}\right) \rightarrow \mathbb{R}$ given by

$$
J(u)=\frac{1}{2} \int_{\mathbb{R}^{N}}|\nabla u|^{2} d x+\frac{1}{2} \int_{\mathbb{R}^{N}} \tilde{V}(x) u^{2} d x+\int_{\mathbb{R}^{N}} u^{2}|\nabla u|^{2} d x-\int_{\mathbb{R}^{N}} G(x, u) d x,
$$

where $G(x, u)=\int_{0}^{u} \widetilde{g}(x, s) d s$ is the primitive of function $\widetilde{g}(x, \cdot)$. However, this functional is not always well defined on all $H^{1}\left(\mathbb{R}^{N}\right)$. We make a change of variables $v:=f^{-1}(u)$, where $f$ is defined by $f^{\prime}(t)=\frac{1}{\sqrt{1+2 f^{2}(t)}}$ on $[0,+\infty)$ and $f(-t)=-f(t)$ on $(-\infty, 0]$.

The following properties of $f$ can be found in [3,7].

Lemma 2.1. The function $f$ satisfies the following properties:

(1) $f$ is uniquely defined, $C^{\infty}$ and invertible;

(2) $\left|f^{\prime}(t)\right| \leq 1$ for all $t \in \mathbb{R}$;

(3) $|f(t)| \leq|t|$ for all $t \in \mathbb{R}$;

(4) $f(t) / 2 \leq t f^{\prime}(t) \leq f(t)$ for all $t>0$;

(5) there exists a positive constant $C_{0}$ such that

$$
|f(t)| \geq \begin{cases}C_{0}|t| & \text { if }|t| \leq 1 \\ C_{0}|t|^{1 / 2} & \text { if }|t| \geq 1\end{cases}
$$

So, after the change of variables from $J$, we obtain the following functional:

$$
I(v):=J(f(v))=\frac{1}{2} \int_{\mathbb{R}^{N}}|\nabla v|^{2} d x+\frac{1}{2} \int_{\mathbb{R}^{N}} \tilde{V}(x) f^{2}(v) d x-\int_{\mathbb{R}^{N}} G(x, f(v)) d x .
$$

At the end of this section, we state the following theorems which are crucial to our arguments in Section 3 .

Let $\Gamma_{k}$ denote the family of closed symmetric subsets $A$ of $E$ such that $0 \notin A$ and the genus $\gamma(A) \geq k$. The following critical point theorem was established in [9].

Lemma 2.2. Let $E$ be an infinite dimensional Banach space and $I \in C^{1}(E, \mathbb{R})$ satisfy $\left(\mathrm{A}_{1}\right)$ and $\left(\mathrm{A}_{2}\right)$ below. 
$\left(\mathrm{A}_{1}\right) I(u)$ is even, bounded from below, $I(0)=0$ and $I(u)$ satisfies the Palais-Smale condition (PS).

$\left(\mathrm{A}_{2}\right)$ For each $k \in \mathbb{N}$, there exists an $A_{k} \in \Gamma_{k}$ such that $\sup _{u \in A_{k}} I(u)<0$.

Then $I(u)$ admits a sequence of critical points $u_{k}$ such that $I\left(u_{k}\right) \leq 0, u_{k} \neq 0$ and $\lim _{k \rightarrow \infty} u_{k}=0$.

\section{Proof of main result}

Let $l=\frac{1}{2} \min \left\{1, \delta_{1}, \delta_{2}\right\}$. We define an even function $h \in C^{1}\left(\mathbb{R}, \mathbb{R}^{+}\right)$such that $0 \leq h(t) \leq 1$, $h(t)=1$ for $|t| \leq l ; h(t)=0$ for $|t| \geq 2 l$ and $h$ is decreasing in $[l, 2 l]$. Let $g_{h}(x, f(v))=$ $\widetilde{g}(x, f(v)) h(f(v))$. Consider the cutoff functional $I_{h}$ :

$$
I_{h}(v)=\frac{1}{2} \int_{\mathbb{R}^{N}}|\nabla v|^{2} d x+\frac{1}{2} \int_{\mathbb{R}^{N}} \widetilde{V}(x) f^{2}(v) d x-\int_{\mathbb{R}^{N}} G_{h}(x, f(v)) d x,
$$

where $G_{h}(x, u)=\int_{0}^{u} g_{h}(x, s) d s$. Then $I_{h}$ is well defined on $E$ and $I_{h} \in C^{1}(E, \mathbb{R})$. The critical points of $I_{h}$ are weak solutions of the problem

$$
-\Delta v+\widetilde{V}(x) f(v) f^{\prime}(v)=g_{h}(x, f(v)) f^{\prime}(v), \quad \forall v \in E .
$$

Then $v \in E$, satisfies $|f(v)| \leq l$, is a critical point of the functional $I_{h}, u=f(v) \in E$ is a weak solution of (1.1).

In order to show that critical points of $I_{h}$ are solutions of (1.1), we will use the following $L^{\infty}$ estimate.

Lemma 3.1. If $\varphi \in E$ is a weak solution of problem (1.1), then $\varphi \in L^{\infty}\left(\mathbb{R}^{N}\right)$. Moreover, there exists $C>0$ such that

$$
|\varphi|_{\infty, \mathbb{R}^{N}} \leq C\|\varphi\|
$$

Proof. For every $\varphi \in E$ and $T>0$, define $\varphi^{T}=\varphi$ for $|\varphi| \leq T, \varphi^{T}=T$ for $\varphi \geq T$ and $\varphi^{T}=-T$ for $\varphi \leq-T$.

Since $I_{h}^{\prime}(\varphi)=0$, we have

$$
\int_{\mathbb{R}^{N}} \nabla \varphi \nabla w d x+\int_{\mathbb{R}^{N}} \tilde{V}(x) f(\varphi) f^{\prime}(\varphi) w d x-\int_{\mathbb{R}^{N}} g_{h}(x, f(\varphi)) f^{\prime}(\varphi) w d x=0
$$

for all $w \in E$.

By $\left(\mathrm{G}_{3}\right)$, the definition of $h$ and Lemma 2.1 $(2)$, we obtain

$$
\left|g_{h}(x, f(\varphi))\right| \leq b(x)|f(\varphi)|^{s}+V_{0}|f(\varphi)| \leq b(x)|\varphi|^{s}+V_{0}|\varphi|
$$

For every $\varphi \in E$, there are two possibilities: (i) $b(x)|\varphi|^{s} \geq V_{0}|\varphi|$; (ii) $b(x)|\varphi|^{s} \leq V_{0}|\varphi|$. 
We first assume that (i) holds. From Lemma 2.1(4), we deduce that $t f(t)>0, \forall t \neq 0$. Taking $w=\varphi\left|\varphi^{T}\right|^{2 r}, r \geq 0$ as a test function in $(3.2)$, we have

$$
\begin{aligned}
& \int_{\mathbb{R}^{N}} g_{h}(x, f(\varphi)) f^{\prime}(\varphi) \varphi\left|\varphi^{T}\right|^{2 r} d x \\
= & \int_{\mathbb{R}^{N}} \nabla \varphi \nabla\left(\varphi\left|\varphi^{T}\right|^{2 r}\right) d x+\int_{\mathbb{R}^{N}} \tilde{V}(x) f(\varphi) f^{\prime}(\varphi) \varphi\left|\varphi^{T}\right|^{2 r} d x \\
\geq & \int_{\mathbb{R}^{N}} \nabla \varphi\left(\nabla \varphi\left|\varphi^{T}\right|^{2 r}+2 r \varphi\left|\varphi^{T}\right|^{2 r-2} \varphi^{T} \nabla \varphi^{T}\right) d x \\
= & \int_{\mathbb{R}^{N}}|\nabla \varphi|^{2}\left|\varphi^{T}\right|^{2 r} d x+2 r \int_{|\varphi| \leq T}\left|\varphi^{T}\right|^{2 r}\left|\nabla \varphi^{T}\right|^{2} d x \\
= & \int_{\mathbb{R}^{N}}|\nabla \varphi|^{2}\left|\varphi^{T}\right|^{2 r} d x+2 r \int_{\mathbb{R}^{N}}\left|\varphi^{T}\right|^{2 r}\left|\nabla \varphi^{T}\right|^{2} d x \\
\geq & \frac{2}{r+2} \int_{\mathbb{R}^{N}}\left(|\nabla \varphi|^{2}\left|\varphi^{T}\right|^{2 r}+2 r\left|\varphi^{T}\right|^{2 r}\left|\nabla \varphi^{T}\right|^{2}+r^{2}\left|\varphi^{T}\right|^{2 r}\left|\nabla \varphi^{T}\right|^{2}\right) d x \\
\geq & \frac{1}{(1+r)^{2}} \int_{\mathbb{R}^{N}}\left|\nabla\left(\varphi\left|\varphi^{T}\right|^{r}\right)\right|^{2} d x \\
\geq & \frac{C}{(1+r)^{2}}\left(\left.\left.\int_{\mathbb{R}^{N}}|\varphi| \varphi^{T}\right|^{r}\right|^{2^{*}} d x\right)^{2 / 2^{*}},
\end{aligned}
$$

where we have used the Gagliardo-Nirenberg-Sobolev inequality. On the other hand, by (3.3) and the Hölder's inequality, one has

$$
\begin{aligned}
& \int_{\mathbb{R}^{N}} g_{h}(x, \varphi) f^{\prime}(\varphi) \varphi\left|\varphi^{T}\right|^{2 r} d x \leq 2 \int_{\mathbb{R}^{N}} b(x)|\varphi|^{s} \varphi\left|\varphi^{T}\right|^{2 r} d x \leq 2 \int_{\mathbb{R}^{N}} b(x)|\varphi|^{2 r+s+1} d x \\
\leq & 2\left(\int_{\mathbb{R}^{N}}|b(x)|^{2 /(1-s)} d x\right)^{(1-s) / 2}\left(\int_{\mathbb{R}^{N}}|\varphi|^{2(2 r+s+1) /(1+s)} d x\right)^{(1+s) / 2} \\
= & 2|b(x)|_{2 /(1-s), \mathbb{R}^{N}}|\varphi|_{2(2 r+s+1) /(1+s), \mathbb{R}^{N}}^{2 r+s+1} .
\end{aligned}
$$

Taking $T \rightarrow \infty$, by $(3.4)$ and $(3.5)$ we have

$$
|\varphi|_{(r+1) 2^{*}, \mathbb{R}^{N}} \leq(C(r+1))^{1 /(r+1)}\left(|\varphi|_{2(2 r+s+1) /(1+s), \mathbb{R}^{N}}^{(2 r+s+1) / 2(r+1)}\right) .
$$

Let $r_{0}=0$ and $2^{*}\left(r_{k}+1\right)=2\left(2 r_{k+1}+s+1\right) /(1+s)$, that is $r_{k}=\frac{\left(2^{*}-2\right)(1+s)}{2^{*}(1+s)-4}\left(\frac{2^{*}(1+s)}{4}\right)^{k}-$ $\frac{\left(2^{*}-2\right)(1+s)}{2^{*}(1+s)-4}$, where $2^{*}(1+s) / 4>1$ since $N=3,4$. Note that $\left(C\left(r_{i}+1\right)\right)^{1 /\left(r_{j}+1\right)} \leq C\left(r_{i}+1\right)$, $\forall i<j$. By iteration we obtain

$$
|\varphi|_{\left(r_{k}+1\right) 2^{*}, \mathbb{R}^{N}} \leq e^{\zeta_{k}}|\varphi|_{2, \mathbb{R}^{N}}^{\sigma_{k}}
$$

where $\zeta_{k}=\sum_{i=0}^{k} \frac{\ln \left[C\left(r_{i}+1\right)\right]}{r_{i}+1}$ and $\sigma_{k}=\prod_{i=0}^{k} \frac{2 r_{i}+s+1}{2\left(r_{i}+1\right)}$, which are convergent as $k \rightarrow \infty$. Let $\zeta=\lim _{k \rightarrow \infty} \zeta_{k}$ and $\sigma=\lim _{k \rightarrow \infty} \sigma_{k}$. Taking the limit as $k \rightarrow \infty$ we obtain that

$$
|\varphi|_{\infty} \leq e^{\zeta}|\varphi|_{2, \mathbb{R}^{N}}^{\sigma} \leq C\|\varphi\|^{\sigma} .
$$


If (ii) holds, without loss of generality, we can assume that $|\varphi| \geq 1$. By (3.3), there holds $\left|g_{h}(x, f(\varphi))\right| \leq 2 V_{0}|\varphi| \leq 2 V_{0}|\varphi|^{q}$, where $q \in\left(2,2^{*}\right)$. Then we can complete the proof by using the well-known Moser iteration.

Lemma 3.2. $I_{h}$ is bounded from below and satisfies the (PS) condition.

Proof. By $\left(\mathrm{G}_{3}\right)$, the definition of $h$ and Lemma 2.1 (3), we have

$$
\left|G_{h}(x, f(v))\right| \leq b(x)|v|^{s+1}+V_{0}|v|^{2}, \quad \forall(x, v) \in\left(\mathbb{R}^{N}, \mathbb{R}\right) .
$$

For any given $v \in E$. Let $\Omega=\left\{x \in \mathbb{R}^{N}:|v| \leq 1\right\}$. By (3.6), Lemma 2.1(3)(5) and Hölder's inequality, one has

$$
\begin{aligned}
I_{h}(v) & =\frac{1}{2} \int_{\mathbb{R}^{N}}|\nabla v|^{2} d x+\frac{1}{2} \int_{\mathbb{R}^{N}} \tilde{V}(x) f^{2}(v) d x-\int_{\mathbb{R}^{N}} G_{h}(x, f(v)) d x \\
& =\frac{1}{2} \int_{\mathbb{R}^{N}}|\nabla v|^{2} d x+\frac{1}{2} \int_{\mathbb{R}^{N}} \tilde{V}(x) f^{2}(v) d x-\int_{\Omega} G_{h}(x, f(v)) d x \\
& \geq \frac{1}{2} \int_{\Omega}|\nabla v|^{2} d x+\frac{C_{0}}{2} \int_{\Omega} \tilde{V}(x) v^{2} d x-\int_{\Omega}\left(b(x)|v|^{s+1}+V_{0}|v|^{2}\right) d x \\
& \geq \frac{1}{2} \int_{\Omega}|\nabla v|^{2} d x+\frac{C_{0}}{2} \int_{\Omega} \tilde{V}(x) v^{2} d x-\int_{\Omega}\left(b(x)|v|^{s+1}+V_{0}|v|^{s+1}\right) d x \\
& \geq C\|v\|_{\Omega}^{2}-|b|_{2 /(1-s), \Omega} \tau_{1+s}^{1+s}\|v\|_{\Omega}^{1+s}-V_{0} \tau_{1+s}^{1+s}\|v\|_{\Omega}^{1+s} \\
& \geq C\|v\|_{\Omega}^{2}-|b|_{2 /(1-s), \mathbb{R}^{N}} \tau_{1+s}^{1+s}\|v\|_{\Omega}^{1+s}-V_{0} \tau_{1+s}^{1+s}\|v\|_{\Omega}^{1+s} .
\end{aligned}
$$

This implies $I_{h}$ is bounded from below.

Next, we prove that $I_{h}$ satisfies the (PS) condition. Let $\left\{v_{n}\right\} \subset E$ be any (PS) sequence of $I_{h}$, i.e., $\left\{I_{h}\left(v_{n}\right)\right\}$ is bounded and $I_{h}^{\prime}\left(v_{n}\right) \rightarrow 0$ in $E^{*}$.

For each $n \in \mathbb{N}$, set $\Omega_{n}=\left\{x \in \mathbb{R}^{N}:\left|v_{n}\right| \leq 1\right\}$. Then by (3.7), we have

$$
C \geq I_{h}\left(v_{n}\right) \geq C\left\|v_{n}\right\|_{\Omega_{n}}^{2}-|b|_{2 /(1-s), \mathbb{R}^{N}} \tau_{1+s}^{1+s}\left\|v_{n}\right\|_{\Omega_{n}}^{1+s}-V_{0} \tau_{1+s}^{1+s}\|v\|_{\Omega_{n}}^{1+s} .
$$

This implies that $\left\|v_{n}\right\|_{\Omega_{n}} \leq C$ and $C$ is independent of $n$. Thus,

$$
\begin{aligned}
& \frac{1}{2} \int_{\Omega_{n}}\left|\nabla v_{n}\right|^{2} d x+\frac{1}{2} \int_{\Omega_{n}} \tilde{V}(x) f^{2}\left(v_{n}\right) d x \\
\leq & I_{h}\left(v_{n}\right)+\int_{\Omega_{n}} G_{h}\left(x, f\left(v_{n}\right)\right) d x \\
\leq & C+|b|_{2 /(1-s), \Omega_{n}} \tau_{1+s}^{1+s}\left\|v_{n}\right\|_{\Omega_{n}}^{1+s}+V_{0} \tau_{1+s}^{1+s}\left\|v_{n}\right\|_{\Omega_{n}}^{1+s} \\
\leq & C+|b|_{2 /(1-s), \mathbb{R}^{N}} \tau_{1+s}^{1+s}\left\|v_{n}\right\|_{\Omega_{n}}^{1+s}+V_{0} \tau_{1+s}^{1+s}\left\|v_{n}\right\|_{\Omega_{n}}^{1+s} \\
\leq & C,
\end{aligned}
$$

where $C$ is independent of $n$. Similarly,

$$
\begin{aligned}
I_{h}\left(v_{n}\right) & =\frac{1}{2} \int_{\mathbb{R}^{N}}\left|\nabla v_{n}\right|^{2} d x+\frac{1}{2} \int_{\mathbb{R}^{N}} \tilde{V}(x) f^{2}\left(v_{n}\right) d x-\int_{\Omega_{n}} G_{h}\left(x, f\left(v_{n}\right)\right) d x \\
& \geq \frac{1}{2} \int_{\mathbb{R}^{N} \backslash \Omega_{n}}\left|\nabla v_{n}\right|^{2} d x+\frac{1}{2} \int_{\mathbb{R}^{N} \backslash \Omega_{n}} \tilde{V}(x) f^{2}\left(v_{n}\right) d x-\int_{\Omega_{n}} G_{h}\left(x, f\left(v_{n}\right)\right) d x .
\end{aligned}
$$


Therefore,

$$
\begin{aligned}
& \frac{1}{2} \int_{\mathbb{R}^{N} \backslash \Omega_{n}}\left|\nabla v_{n}\right|^{2} d x+\frac{1}{2} \int_{\mathbb{R}^{N} \backslash \Omega_{n}} \tilde{V}(x) f^{2}\left(v_{n}\right) d x \leq I_{h}\left(v_{n}\right)+\int_{\Omega_{n}} G_{h}\left(x, f\left(v_{n}\right)\right) d x \\
\leq & C+|b|_{2 /(1-s), \mathbb{R}^{N}} \tau_{1+s}^{1+s}\left\|v_{n}\right\|_{\Omega_{n}}^{1+s}+V_{0} \tau_{1+s}^{1+s}\left\|v_{n}\right\|_{\Omega_{n}}^{1+s} \leq C,
\end{aligned}
$$

where $C$ is independent of $n$. Then from (3.8) and (3.9), we get that $D_{n}^{2}:=\int_{\mathbb{R}^{N}}\left|\nabla v_{n}\right|^{2} d x+$ $\int_{\mathbb{R}^{N}} \tilde{V}(x) f^{2}\left(v_{n}\right) d x$ is bounded independent of $n$.

From [16, we know that there exists a constant $C>0$ such that $D_{n}^{2} \geq C\left\|v_{n}\right\|^{2}$. Then $\left\{v_{n}\right\}$ is bounded in $E$ by the above arguments. Thus, up to a subsequence, we have $v_{n} \rightarrow v$ in $E, v_{n} \rightarrow v$ in $L^{p}\left(\mathbb{R}^{N}\right)$ for $2 \leq p<2^{*}$ and $v_{n} \rightarrow v$ a.e. on $\mathbb{R}^{N}$. By $\left(\mathrm{G}_{3}\right)$ and Lemma 2.1 (2)(3), one has

$$
\begin{aligned}
& \left|\int_{\mathbb{R}^{N}}\left(g_{h}\left(x, f\left(v_{n}\right)\right) f^{\prime}\left(v_{n}\right)-g_{h}(x, f(v)) f^{\prime}(v)\right)\left(v_{n}-v\right) d x\right| \\
\leq & \int_{\mathbb{R}^{N}}\left(|b(x)|\left|v_{n}\right|^{s}+V_{0}\left|v_{n}\right|+|b(x)||v|^{s}+V_{0}|v|\right)\left|\left(v_{n}-v\right)\right| d x \\
\leq & \left(|b|_{2 /(1-s), \mathbb{R}^{N}}\left|v_{n}\right|_{2, \mathbb{R}^{N}}^{s}+V_{0}\left|v_{n}\right|_{2, \mathbb{R}^{N}}+|b|_{2 /(1-s), \mathbb{R}^{N}}|v|_{2, \mathbb{R}^{N}}^{s}+V_{0}|v|_{2, \mathbb{R}^{N}}\right)\left|v_{n}-v\right|_{2, \mathbb{R}^{N}} \\
= & o_{n}(1) .
\end{aligned}
$$

As in the proof of Lemma 3.1 in [7] (see also Theorem 2.1 in [16]), we may prove that there exists a constant $C>0$ such that

$$
\int_{\mathbb{R}^{N}}\left|\nabla\left(v_{n}-v\right)\right|^{2} d x+\int_{\mathbb{R}^{N}} \tilde{V}(x)\left(f\left(v_{n}\right) f^{\prime}\left(v_{n}\right)-f(v) f^{\prime}(v)\right)\left(v_{n}-v\right) d x \geq C\left\|v_{n}-v\right\|^{2} .
$$

By 3.10 and (3.11), we have

$$
\begin{aligned}
o_{n}(1)= & \left\langle I_{h}^{\prime}\left(v_{n}\right)-I_{h}^{\prime}(v), v_{n}-v\right\rangle \\
= & \int_{\mathbb{R}^{N}}\left|\nabla\left(v_{n}-v\right)\right|^{2} d x+\int_{\mathbb{R}^{N}} \widetilde{V}(x)\left(f\left(v_{n}\right) f^{\prime}\left(v_{n}\right)-f(v) f^{\prime}(v)\right)\left(v_{n}-v\right) d x \\
& -\int_{\mathbb{R}^{N}}\left(g_{h}\left(x, f\left(v_{n}\right)\right) f^{\prime}\left(v_{n}\right)-g_{h}(x, f(v)) f^{\prime}(v)\right)\left(v_{n}-v\right) d x \\
\geq & C\left\|v_{n}-v\right\|^{2}+o_{n}(1) .
\end{aligned}
$$

Hence $v_{n} \rightarrow v$ in $E$. The proof is completed.

In order to prove Theorem 1.1, we shall use a similar argument of [17].

Lemma 3.3. For any $n \in \mathbb{N}$, there exist closed symmetric subsets $A_{n} \subset E$ such that the genus $\gamma\left(A_{n}\right) \geq n$ and $\sup _{v \in A_{n}} I_{h}(v)<0$. 
Proof. Let $E_{n}$ be any $n$-dimensional subspace of $E$. Since all norms are equivalent in a finite dimensional space, there is a constant $\tau=\tau\left(E_{n}\right)$ such that

$$
\|v\| \leq \tau|v|_{2}
$$

for all $v \in E_{n}$.

Claim: there exists a constant $\kappa>0$ such that

$$
\frac{1}{2} \int_{\mathbb{R}^{N}}|v|^{2} d x \geq \int_{|f(v)|>l}|v|^{2} d x
$$

for all $v \in E_{n}$ with $\|v\| \leq \kappa$. Indeed, if 3.12 is false, there exists a sequence $\left\{v_{k}\right\} \subset$ $E_{n} \backslash\{0\}$ such that $v_{k} \rightarrow 0$ in $E$ and

$$
\frac{1}{2} \int_{\mathbb{R}^{N}}\left|v_{k}\right|^{2} d x<\int_{\left|f\left(v_{k}\right)\right|>l}\left|v_{k}\right|^{2} d x, \quad k \in \mathbb{N} .
$$

Let $u_{k}=v_{k} /\left|v_{k}\right|_{2}$. Then

$$
\frac{1}{2}<\int_{\left|f\left(v_{k}\right)\right|>l}\left|u_{k}\right|^{2} d x, \quad k \in \mathbb{N} .
$$

On the other hand, we can assume that $u_{k} \rightarrow u$ in $E$ since $E_{n}$ is finite dimensional. Hence $u_{k} \rightarrow u$ in $L^{2}\left(\mathbb{R}^{N}\right)$. Moreover, it can be deduced from $v_{k} \rightarrow 0$ in $E$ that

$$
\operatorname{meas}\left\{x \in \mathbb{R}^{N}:\left|f\left(v_{k}\right)\right|>l\right\} \rightarrow 0, \quad k \rightarrow \infty .
$$

Therefore,

$$
\int_{\left|f\left(v_{k}\right)\right|>l}\left|u_{k}\right|^{2} d x \leq 2 \int_{\mathbb{R}^{N}}\left|u_{k}-u\right|^{2} d x+2 \int_{\left|f\left(v_{k}\right)\right|>l}|u|^{2} d x \rightarrow 0, \quad k \rightarrow \infty,
$$

which contradicts $(3.13)$ and hence 3.12 holds.

By $\left(\mathrm{G}_{2}\right)$, we can choose $l$ small enough such that

$$
g(x, v) \geq 4 \tau^{2} v
$$

for all $x \in \mathbb{R}^{N}$ and $0 \leq v \leq 2 l$. This inequality implies that

$$
G_{h}(x, v)=G(x, v) \geq 2 \tau^{2} v^{2}, \quad \forall(x, v) \in \mathbb{R}^{N} \times[0, l] .
$$

The assumption $\left(\mathrm{G}_{1}\right)$ implies $G_{h}(x, v)$ is even in $v$. Thus, by (3.14), we have

$$
\begin{aligned}
I_{h}(v) & \leq \frac{1}{2}\|v\|^{2}-\int_{\mathbb{R}^{N}} G_{h}(x, f(v)) d x \leq \frac{1}{2}\|v\|^{2}-\int_{|f(v)| \leq l} G_{h}(x, f(v)) d x \\
& \leq \frac{1}{2}\|v\|^{2}-2 \tau^{2} \int_{|f(v)| \leq l}|v|^{2} d x \leq \frac{1}{2}\|v\|^{2}-2 \tau^{2}\left(\int_{\mathbb{R}^{N}}|v|^{2} d x-\int_{|f(v)|>l}|v|^{2} d x\right) \\
& \leq-\frac{1}{2}\|v\|^{2}
\end{aligned}
$$


for all $v \in E_{n}$ with $\|v\| \leq \kappa$.

Let $0<\rho \leq \kappa$ and $A_{n}=\left\{v \in E_{n}:\|v\|=\rho\right\}$. We conclude that $\gamma\left(A_{n}\right) \geq n$ and

$$
\sup _{v \in A_{n}} I_{h}(v) \leq-\frac{1}{2} \rho^{2}<0 .
$$

The proof is completed.

Proof of Theorem 1.1. By $\left(\mathrm{G}_{1}\right)$ and $\left(\mathrm{G}_{3}\right)$, we get that $I_{h}$ is even and $I_{h}(0)=0$. Then from Lemmas 2.2, 3.2 and 3.3 we obtain that $I_{h}$ has a critical sequence $\left\{v_{n}\right\}$ converging to 0. By Lemma 3.1. we obtain that $v_{n} \in L^{\infty}\left(\mathbb{R}^{N}\right)$. Moreover, from (3.1), we may get that there exists $n_{1}$ such that $\left|v_{n}\right|_{\infty, \mathbb{R}^{N}} \leq l$ for $n \geq n_{1}$. Then we get infinitely many small solutions of (1.1). The proof is completed.

\section{References}

[1] A. de Bouard, N. Hayashi and J.-C. Saut, Global existence of small solutions to a relativistic nonlinear Schrödinger equation, Comm. Math. Phys. 189 (1997), no. 1, 73-105.

[2] L. Brüll and H. Lange, Solitary waves for quasilinear Schrödinger equations, Exposition. Math. 4 (1986), no. 3, 279-288.

[3] M. Colin and L. Jeanjean, Solutions for a quasilinear Schrödinger equation: a dual approach, Nonlinear Anal. 56 (2004), no. 2, 213-226.

[4] J. M. B. do Ó, O. H. Miyagaki and S. H. M. Soares, Soliton solutions for quasilinear Schrödinger equations with critical growth, J. Differential Equations. 248 (2010), no. $4,722-744$.

[5] J. M. do Ó and U. Severo, Quasilinear Schrödinger equations involving concave and convex nonlinearities, Commun. Pure Appl. Anal. 8 (2009), no. 9, 621-644.

[6] Solitary waves for a class of quasilinear Schrödinger equations in dimension two, Calc. Var. Partial Differential Equations 38 (2010), no. 3-4, 275-315.

[7] X.-D. Fang and A. Szulkin, Multiple solutions for a quasilinear Schrödinger equation, J. Differential Equations 254 (2013), no. 4, 2015-2032.

[8] E. Gloss, Existence and concentration of positive solutions for a quasilinear equation in $\mathbb{R}^{N}$, J. Math .Anal. Appl. 371 (2010), no. 2, 465-484.

[9] R. Kajikiya, A critical point theorem related to the symmetric mountain pass lemma and its applications to elliptic equations, J. Funct. Anal. 225 (2005), no. 2, 352-370. 
[10] Z. Liang, J. Gao and A. Li, Infinitely many solutions to a quasilinear Schrödinger equation with a local sublinear term, Appl. Math. Lett. 89 (2019), 22-27.

[11] J. Liu and Z.-Q. Wang, Soliton solutions for quasilinear Schrödinger equations I, Proc. Amer. Math. Soc. 131 (2003), no. 2, 441-448.

[12] J.-q. Liu, Y.-q. Wang and Z.-Q. Wang, Soliton solutions for quasilinear Schrödinger equations II, J. Differential Equations 187 (2003), no. 2, 473-493.

[13] Solutions for quasilinear Schrödinger equations via the Nehari method, Comm. Partial Differential Equations 29 (2004), no. 5-6, 879-901.

[14] J.-Q. Liu, Z.-Q. Wang and Y.-X. Guo, Multibump solutions for quasilinear elliptic equations, J. Funct. Anal. 262 (2012), no. 9, 4040-4102.

[15] M. Poppenberg, K. Schmitt and Z.-Q. Wang, On the existence of soliton solutions to quasilinear Schrödinger equations, Calc. Var. Partial Differential Equations 14 (2002), no. 3, 329-344.

[16] X. Wu, Multiple solutions for quasilinear Schrödinger equations with a parameter, J. Differential Equations 256 (2014), no. 7, 2619-2632.

[17] F. Zhou and K. Wu, Infinitely many small solutions for a modified nonlinear Schrödinger equations, J. Math. Anal. Appl. 411 (2014), no. 2, 953-959.

[18] W. Zou and M. Schechter, Critical Point Theory and its Applications, Springer, New York, 2006.

Gui Bao and Tingzhi Cheng

School of Mathematics and Statistics Science, Ludong University, Yantai, Shandong 264025, China

E-mail address: baoguigui@163.com, chengtingzhi1989@163.com 